\section{The Lymph Node Ratio Staging System in Gastric Cancer}

\section{TO THE EDITORS:}

The article by Esther (ASO-2009-09-1193) examined the prognostic significance of the lymph node ratio (LNR) between metastatic and examined lymph nodes for recurrence and survival in gastric cancer. The finding is similar to the recent studies by Sun et al. and Kulig et al. ${ }^{1,2}$ They consider that the LNR is a simple and reliable prognostic factor in resected gastric cancer patients with insufficient nodes retrieved.

In previous studies, many researchers have proposed the prognostic significance of the LNR in minimizing stagemigration phenomenon and predicting patient survival for gastric patients with $>15$ examined lymph nodes. Moreover, Italian multicentric study suggests that LNR is a better prognostic factor as compared with traditional staging systems regardless of the type of lymphadenentomy. ${ }^{3}$ Our study also identified positive lymph node ratio as an independent prognostic factor after D2 resection regardless of the number of lymph nodes examined. ${ }^{4}$ These results make it possible that a staging system based on the LNR (TRM) could substitute for the current International Union against Cancer (UICC) classification used most widely for gastric cancer.

However, this does not mean that we can neglect our work on searching for lymph nodes. For example, when no metastatic lymph nodes are found among patients with insufficient nodes retrieved, it is difficult to evaluate the prognosis of gastric cancer patients by TRM. In fact, in different type of lymphadenectomy, a minimal number of retrieved nodes could be essential to guarantee the potential ability of TRM to minimize stage-migration phenomenon. ${ }^{1}$

On the other hand, some published studies have reported different LNR cutoffs. In order to make the study objectively valid, we examined the functional form of the covariate by Martingale residual analysis and identified the cutoff value using the log-rank test. ${ }^{4}$ The optimum cutoff value was also estimated using reverse Helmert contrasts. ${ }^{2}$ However, this article by Esther classified the LNR cutoff without applying any specific method for their selection.

Indeed, there is always disagreement over the use of staging standards based on the LNR cutoff. To determine the optimal cutoff value for the LNR, it may be necessary to further analyze large-scale data from multicentre settings.

\section{Da-zhi Xu, MD, PhD ${ }^{1,2}$}

${ }^{1}$ State Key Laboratory of Oncology in South China, Cancer Center, Sun Yat-sen University, Guangzhou, China;

${ }^{2}$ Department of Abdominal Surgery, Cancer Center, Sun Yat-sen University, Guangzhou, China

e-mail:xdz1976@yahoo.com.cn

Published Online: 4 November 2009

(C) Society of Surgical Oncology 2009

\section{REFERENCES}

1. Sun Z, Zhu GL, Lu C, Guo PT, Huang BJ, Li K, et al. The impact of $\mathrm{N}$-ratio in minimizing stage migration phenomenon in gastric cancer patients with insufficient number or level of lymph node retrieved: results from a Chinese mono-institutional study in 2159 patients. Ann Oncol. 2009;20(5):897-905.

2. Kulig J, Sierzega M, Kolodziejczyk P, Popiela T, Polish Gastric Cancer Study Group. Ratio of metastatic to resected lymph nodes for prediction of survival in patients with inadequately staged gastric cancer. Br J Surg. 2009;96(8):910-8.

3. Marchet A, Mocellin S, Ambrosi A, Morgagni P, Garcea D, Marrelli D, et al. The ratio between metastatic and examined lymph nodes ( $\mathrm{N}$ ratio) is an independent prognostic factor in gastric cancer regardless of the type of lymphadenectomy. Ann Surg. 2007;245(4):543-52.

4. Xu DZ, Geng QR, Long ZJ, Zhan YQ, Li W, Zhou ZW, et al. Positive lymph node ratio is an independent prognostic factor in gastric cancer after D2 resection regardless of the examined number of lymph nodes. Ann Surg Oncol. 2009;16(2):319-26. 